\title{
Quantitative determination of local potential values in inhomogeneously doped semiconductors by scanning tunneling microscopy
}

\author{
P. H. Weidlich, R. E. Dunin-Borkowski, and Ph. Ebert ${ }^{*}$ \\ Peter Grünberg Institut, Forschungszentrum Jülich GmbH, 52425 Jülich, Germany
}

(Received 5 April 2011; published 29 August 2011)

\begin{abstract}
Local potential changes arising from nanoscale three-dimensional spatial fluctuations in the dopant distribution in Zn-doped GaAs were investigated quantitatively by scanning tunneling microscopy and spectroscopy at (110) cleavage surfaces. Tunneling spectra measured in areas with different local doping concentration show apparent shifts of the valence band edge and apparent changes of the band gap. A quantitative analysis, combined with band bending and tunnel current simulations, demonstrates that these effects arise from tip-induced band bending that modulates the real potential changes. It is illustrated how exact potential changes between locally high and low doped areas can be determined. It is found that the local potential fluctuations in three-dimensionally doped semiconductors are approximately one order of magnitude smaller that those observed in two-dimensionally doped semiconductors.
\end{abstract}

DOI: 10.1103/PhysRevB.84.085210 PACS number(s): 71.55.Eq, 68.37.Ef, 41.20.Cv, 73.20.Hb

\section{INTRODUCTION}

With the ongoing push for faster electronics with higher performances, the electrically active device regions increasingly shrink, ultimately reaching dimensions where the device operation depends on few or even single electrons. In such nanoscale devices, fluctuations in the dopant distribution have a crucial impact on the physical properties of the devices and

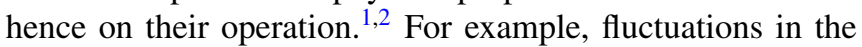
spatial distribution of dopant atoms ${ }^{3}$ influence the threshold voltages by causing nano- and atomic-scale fluctuations in the potential. ${ }^{4,5}$ Thus, it is critical to quantitatively probe the local potential at nano- and atomic scales and relate it to the local dopant distribution. In this manner, quantitative physical models can be derived for the potential in spatially and dimensionally reduced semiconductor devices. ${ }^{4}$ Therefore, in recent years intensive efforts were made to experimentally probe the local potential with the highest possible spatial and energetic resolution in semiconductors using a variety of experimental approaches.

First, electron holography in a transmission electron microscope (TEM) has been applied to determine the potential, thus far, primarily of $p-n$ junctions. ${ }^{6-9}$ Twitchett-Harrison et al. demonstrated that, in combination with a tomographic reconstruction, quantitative three-dimensional potential values across $p$ - $n$ junctions can be derived by measuring the phase change of the high-energy electrons passing the specimen. ${ }^{10,11}$ Although quantitative potential values can be derived for the bulk, no atomic resolution is yet possible and surface effects, as well as irradiation damage, still play a role. A determination of the positions of individual dopants using TEM can be achieved at present with atomic column resolution ${ }^{12}$ but without simultaneous potential measurements.

Second, scanning probe techniques were used to probe the local potential at surfaces. To probe values representing the potential in the bulk, the surface needs to be free of surface states in the fundamental band gap. This is the case for most compound semiconductor cleavage surfaces, as well as for passivated Si surfaces. On such surfaces, Kelvin probe force microscopy allows the measurement of the local potential, e.g., at surface steps with a $10-\mathrm{meV}$ energy resolution. ${ }^{13}$ However, atomic resolution is not yet achieved. In contrast, atomic resolution combined with high local energy resolution can be routinely achieved using scanning tunneling microscopy (STM). For example, the band offsets between different semiconductor materials ${ }^{14}$ or the potential distribution at $p-n$ junctions ${ }^{15,16}$ could be probed. The local potential can also be directly imaged with atomic resolution as shown, e.g., for the screened Coulomb potentials around charged point defects or steps, ${ }^{17-21}$ potential fluctuations due to inhomogeneous dopant ${ }^{4,5}$ or adatom distributions, ${ }^{22,23}$ or epitaxial structures. ${ }^{5,24,25}$ Despite intensive efforts on potential measurements by STM, only a limited number of quantitative measurements exist. The frequently assumed proportionality of the corrugation changes and the potential is not valid, as demonstrated in Ref. 26. Quantitative potential measurements rely on a statistical analysis of interacting charged defects, ${ }^{19,20,26}$ the band edge determination in tunneling spectra, ${ }^{4,14}$ a work function measurement, or surface photo voltage measurements. ${ }^{27}$ However, in the latter two cases, the tip-induced band bending severely affects the results by shifting the band edges. ${ }^{28-31}$ This band edge shift is not homogeneous across the surface, because the screening ability varies spatially, e.g., with the fluctuations in the dopant distribution or the material across heterointerfaces. In addition, the tip-induced band bending can lead to charge carrier accumulations, which increase the screening ability, reducing the screening length and thereby the spatial extension of the screened Coulomb potential artificially. ${ }^{26}$ Thus far, a quantitative reliable determination of the band edge positions from tunneling spectra and the potential distribution from constant-current STM images was only possible for a two-dimensional semiconductor, where due to the extremely high two-dimensional carrier concentration, no tip-induced band bending is present. ${ }^{4}$ However, even in the presence of tip-induced band bending, the STM data inherently include information about the local potential. Therefore, it is highly desirable to derive a methodology on how to determine the local potential from tunneling spectra in the presence of tip-induced band bending. 
In this paper, we experimentally probe tunneling spectra in areas with different local doping concentrations due to threedimensional spatial fluctuations in the dopant distribution in our model system, Zn-doped GaAs. The tunneling spectra show apparent shifts of the valence band edge and changes of the band gap. A quantitative analysis, combined with band bending and tunnel current simulations, demonstrates that these effects arise from tip-induced band bending that modulates the real potential changes. A methodology is illustrated on how the exact potential changes between locally high and low doped areas can be determined. The thereby obtained potential fluctuations in three-dimensionally doped semiconductors are one order of magnitude smaller than in two-dimensional semiconductors. This is explained by damping effects due to the three-dimensional arrangement of the dopants.

\section{EXPERIMENT}

For the present work, we investigated samples cut from melt-grown, highly $p$-doped GaAs. The zinc dopant concentration is nominally $4 \times 10^{19} \mathrm{~cm}^{-3}$. The samples were cleaved in ultrahigh vacuum $\left(p \approx 1 \times 10^{-8} \mathrm{~Pa}\right)$ using a double wedge cleavage technique to expose a clean and defect-free GaAs(110) surface. On these surfaces, we acquired STM and spectroscopy data at room temperature. With the voltage applied to the sample, the STM images were acquired in the constant-current mode with set voltages and set current as indicated later. The tunneling spectra were measured at fixed tip-sample separations. For the measurements, we used electrochemically etched $\mathrm{W}$ tips.

\section{DETAILS OF THE CALCULATION}

For the analysis of our results, we calculated the positions of the conduction band $\left(E_{\mathrm{CS}}\right)$ and valence band edges $\left(E_{\mathrm{VS}}\right)$ at the GaAs surface underneath the $\mathrm{W}$ tip, as well as the resulting tunneling current. We based our calculation of the band edge energies on the one-dimensional integration of Poisson's equation described by Feenstra and Stroscio, ${ }^{28}$ as well as Seiwatz and Green, ${ }^{32}$ but extended it to include the effects of carrier dynamics. ${ }^{33}$ This is necessary, because the procedure of Refs. (28) and (32) assumes equilibrium conditions for the carrier distribution in the semiconductor. As shown in Ref. 33, this assumption is not valid; therefore, we included the effects of carrier dynamics by setting the minority and/or majority carrier concentration at the surface to zero. The tunneling current is computed exactly as described by Feenstra and Stroscio, ${ }^{28}$ as well as Bono and Good. ${ }^{34}$ This model is based on the approach by Tersoff and Hamann ${ }^{35}$ and Selloni et al., ${ }^{36}$ where the current is obtained by integrating over all states between the tip and the sample Fermi level times the transmission coefficient. Parabolic bands are assumed for the density of valence and conduction band states. The transmission coefficient through the vacuum barrier, as well as through the semiconductor's space charge region, is estimated using the Wentzel-Kramers-Brillouin approximation. Thereby, the vacuum barrier is taken as trapezoidal modified by the image charge lowering.
Our calculations were performed for $\mathrm{Zn}$-doped GaAs with carrier concentrations ranging from $2 \times 10^{18}$ to $8 \times 10^{19}$ $\mathrm{cm}^{-3}$. In all cases, a background donor concentration of $1 \times 10^{14} \mathrm{~cm}^{-3}$, a work function of $4.5 \mathrm{eV}$ for the metallic tungsten tip, a tip-sample separation of $0.9 \mathrm{~nm}$, and a tunneling area of $1 \mathrm{~nm}^{2}$ were assumed. ${ }^{28}$ A variation of these parameters does not change the results qualitatively.

\section{EXPERIMENTAL RESULTS}

Figure 1(a) shows a large-scale constant-current STM image of a freshly in situ cleaved $p$-type GaAs(110) surface. The image shows the occupied density of states acquired at negative sample voltage. ${ }^{37}$ The surface is atomically flat and free of steps. Two types of highly localized features are visible, which can be classified as dark and bright localized spots. A high-resolution image in Fig. 1(b) shows these twotypes of localized contrast, superimposed on the atomic corrugation, which arises from the occupied dangling bonds localized above the As atoms bonded in the zigzag atomic rows of the surface parallel to the [110] direction. ${ }^{37}$ The typically 1 to 2-nm-sized dark features correspond to isolated positively charged anion vacancies, as observed before on (110) surfaces of $p$-type III-V semiconductors. ${ }^{38}$ The atomically localized dark features are uncharged (and thus electrically inactive) anion vacancy-Zn dopant complexes. ${ }^{39}$ Finally, the bright localized features exhibit different types of local symmetries and intensities. We observed an alternating change of the mirror planes between and on top of the maxima along the atomic rows with decreasing brightness. We can discern in this particular case five levels of brightness, as indicated by number in Fig. 1(b). These features are compatible with $\mathrm{Zn}$ dopant atoms in different layers below the cleavage surface. ${ }^{39,40}$ The bright contrast arises from the localized negative charge of the $\mathrm{Zn}$ acceptors, giving rise to a local screened Coulomb potential. ${ }^{17,18}$ The five contrasts of $\mathrm{Zn}$ acceptors indicate that in the present case the STM is sensitive to probe dopant atoms within the top five subsurface layers. This interpretation is corroborated by the observed concentration of bright features. Taking into account that the dopants can be observed up to a depth of five atomic layers, we obtain a dopant concentration of $3 \times 10^{19} \mathrm{~cm}^{-3}$. This is in good agreement with the nominal dopant concentration.

One of the most distinctive features in the STM image in Fig. 1(a) is the long-range height contrast change on the scale of $\sim 10-20 \mathrm{~nm}$ superposed on the localized features of the dopant atoms. The contrast is directly related to the local dopant concentration. A dark contrast is observed in areas without dopant atoms or with a low dopant concentration (e.g., area $\mathrm{A}$ ), whereas a bright contrast occurs in areas with a high dopant concentration (e.g., area B). The fluctuations in the distribution of dopant atoms are typical for $p$-type GaAs and were found to arise from many body effects in the dopantdopant interactions during growth of the GaAs crystal in this specific case. $^{3}$

At this stage, we address the origin of the long-range contrast fluctuations. These fluctuations become more pronounced at lower magnitudes of the voltage, despite the surface being atomically flat and free of mechanical bending or steps. This indicates that the contrast fluctuations are almost exclusively 
(a)

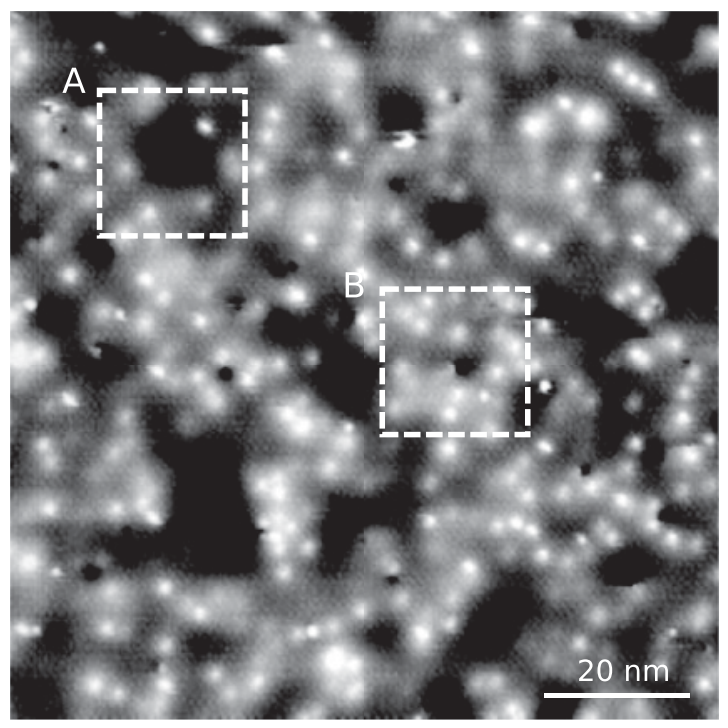

(b)

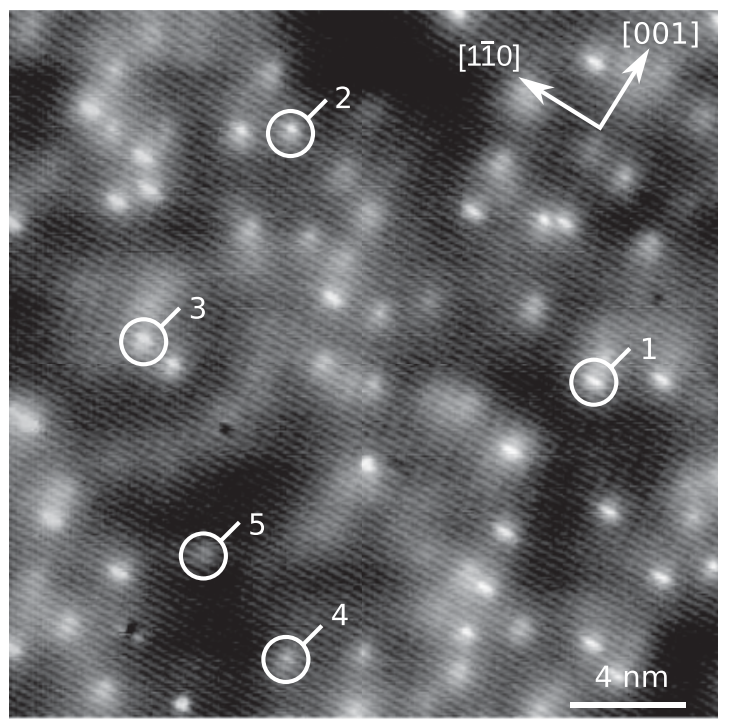

FIG. 1. (a) Overview constant-current STM image of the filled density of states of a nominally $4 \times 10^{19} \mathrm{~cm}^{-3} \mathrm{Zn}$-doped GaAs(110) cleavage surface measured at a sample voltage of $-2.5 \mathrm{~V}$ and a tunnel current of $0.7 \mathrm{nA}$. The bright localized contrasts arise from negatively charged $\mathrm{Zn}_{\mathrm{Ga}}$ acceptors. The localized dark spots are due to anion (As) vacancies. The local dopant concentration exhibits pronounced fluctuations. In some areas, no or only few dopant atoms are present (e.g., the area marked A), whereas in other areas high dopant concentrations exists (e.g., area B). The inhomogeneous dopant distribution leads to long-range fluctuations of the contrast, pointing to local fluctuations of the potential with lateral dimensions of 10-20 nm. (b) High-resolution constant-current STM image measured at $-2.1 \mathrm{~V}$ and $0.7 \mathrm{nA}$. Individual dopant atoms in the first five subsurface layers can be distinguished on the basis of the contrast intensity and symmetry. For each layer, one example is marked. The surface is atomically flat, without any atomic steps. The contrast visible in both STM images is exclusively an electronic contrast arising from local potential fluctuations and not due to any mechanical bending of the surface. Both STM images have the same orientation. of electronic origin. They are the signature of variations of the local potential (times the electron charge $e$ ), i.e., the position of the valence band edge changing locally relative to the Fermi level. ${ }^{4,5,25}$ If the valence band edge is increasingly below the Fermi energy (i.e., less $p$ doped), the number of states available for tunneling out of the filled valence band states decreases. This leads to a smaller tunnel current, which is compensated by the feedback loop in the constant-current image by approaching the tip toward the surface. This reduced tip-sample separation is shown in Fig. 1 as darker contrast.

To quantify the magnitude of local potential fluctuations related to the fluctuations in the dopant distribution, we turn to scanning tunneling spectra (Fig. 2). Figure 2(a) shows two $I-V$ spectra measured in a bright and in a dark area. Each shown spectrum is an average of several spectra to improve the signal-to-noise ratio. These $I-V$ curves were chosen from sets of $I-V$ curves, which were measured at different tipsample separations (not shown here). The different tip-sample separations were adjusted using different set-point tunneling voltages $\left(V_{\text {set }}\right)$ and currents $\left(I_{\text {set }}\right)$, before holding the tip at a certain fixed tip-sample separation and position. At a constant $I_{\text {set }}$, the tip-sample separation increases with $V_{\text {set }}$. Each $I-V$ curve exhibits no detectable current (detection limit of $\sim 1 \mathrm{pA}$ ) in a voltage range of $\sim 0 \mathrm{~V}$ (Fig. 2(a)). The absence of the current indicates the presence of a band gap. However, the transmission coefficient for electron tunneling decreases with tip-sample separation. Hence, the apparent band gap increases and the band edge positions cannot be determined due to insufficient sensitivity of the current measurement. Therefore, we concentrate in Fig. 2 on curves measured at a tip-sample separation that is small enough for a sufficient sensitivity even within the band gap but still well in the tunneling regime, i.e., no point contact. ${ }^{41}$

Another problem causing serious complications in the direct interpretation of the as-measured tunneling spectra are the different tip-sample separations in areas with dark and bright contrast, even for identical tunneling set points. The current is primarily determined by the local electronic structure. Thus, even on atomically flat surfaces, the tip height changes if the underlying surface exhibits local variations in the electronic structure. To allow a direct comparison of the different spectra, we corrected all curves to an identical tip-sample separation by measuring the exponential dependence of the current $I$ as a function of tip-sample distance $z, I=I_{0} \cdot e^{-2 \kappa z}$ (inset in Fig. 2(b)). Using the decay constant $2 \kappa$ determined from an exponential fit to the measured current values (solid line in the inset in Fig. 2(b)) and the height difference between the dark and the bright areas in the constant-current STM images, we recalibrated the tunnel current to one constant tip-sample separation (Fig. 2(b)).

To identify the energetic positions of the band edges relative to the Fermi energy $E_{\mathrm{F}}$, we first need to address the origins of the current. For this, we turn to the logarithmic display of the absolute current as a function of the sample bias (Fig. 2(b)) for the corrected $I-V$ spectra. The logarithmically displayed current curves exhibit a clear single onset $V_{\mathrm{C} \text {,onset }} \approx+1.45 \mathrm{~V}$ of the tunneling current into the empty conduction band states of the surface (labeled $I_{\mathrm{C}}$ ) in both cases. At negative voltages, a single onset $V_{\mathrm{V} \text {,onset }}$ of the tunnel current out of the valence 
(a)

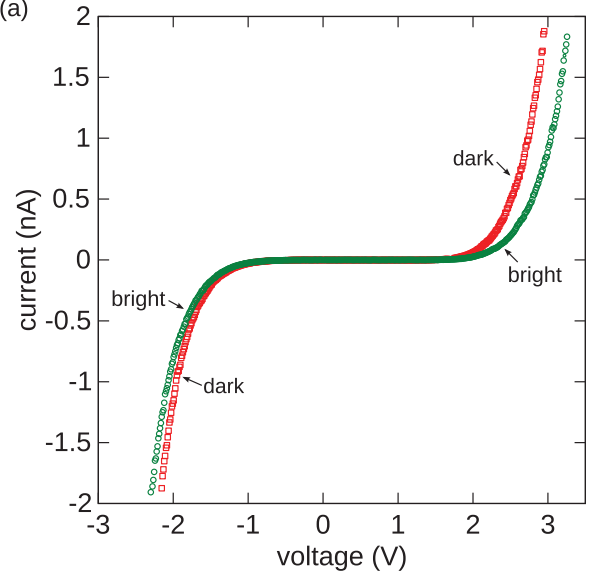

(b)
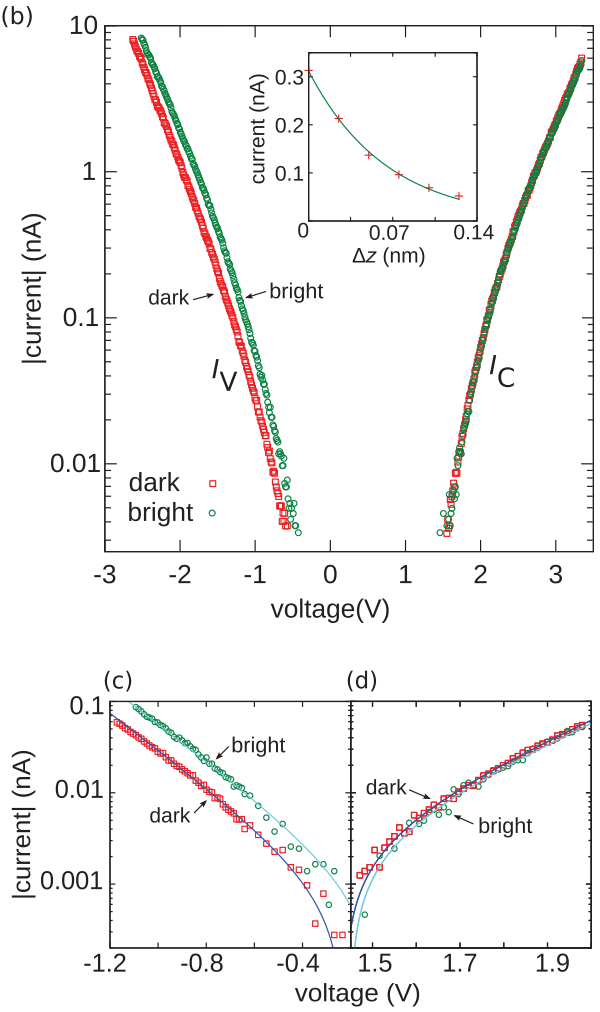

FIG. 2. (Color online) (a) Current-voltage spectra taken in dark (red squares) and bright (green circles) areas, respectively. The spectra shown are average spectra, where each individual spectrum has been measured at a tip-sample separation fixed by a set voltage of $-1.53 \mathrm{~V}$ and a set current of $0.2 \mathrm{nA}$. (b) Although each spectrum in (a) has been measured at a tip-sample separation $z$ determined by the same set voltage and set current, the spectra measured in the dark and bright areas were not measured at the same tip-sample separation $z$ due to the different local potential and thus different density of states contributing to the tunnel current in the dark and bright areas, respectively. As a result, the feedback loop adjusts tip-sample separations to keep the tunnel current constant, leading to the different height contrast in the dark and bright areas. To compare the spectra measured on an atomically flat surface correctly, (b) shows the logarithmic display of the current voltage spectra corrected to the same tip-sample separation using the current-distance $(I-\Delta z)$ measurement shown in the inset, where $\Delta z$ is the change of the tip-sample separation $z$. (c) and (d) show, respectively, the valence and the conduction band currents expanded. band states $\left(I_{\mathrm{V}}\right)$ occurs for both curves too. However, the absolute values of the onset voltages decrease from the dark toward the bright surface area. To quantify the shift of the onset voltages of valence band-related current, a close-up is shown in Fig 2(c). The fits to the data (solid lines) demonstrate a voltage shift of $+(0.14 \pm 0.02) \mathrm{eV}$ from the dark to the bright areas. In contrast, the onset of the conduction band-related current is almost identical for both areas (shift of $0.04 \pm 0.07 \mathrm{eV}$ ), as visible in the close-up shown in Fig. 2(d). The positions of the band edges and the respective onset voltages for tunneling into bands are not affected by the thermal broadening, because the broadening does not shift the bands.

\section{DISCUSSION}

\section{A. Extraction of the local potential}

To extract the local potential $U=E_{\mathrm{V}}-E_{\mathrm{F}}$ from the tunneling spectra, i.e., the position of the valence band edge $\left(E_{\mathrm{V}}\right)$ relative to the Fermi energy $\left(E_{\mathrm{F}}\right)$, we recall that electrons start to tunnel from the sample toward the tip as soon as the valence band edge is at (and above) the Fermi energy of the tip $E_{\mathrm{F}, \text { tip }}$. Similarly, electrons tunnel into the conduction band once the conduction band edge is at or below the Fermi energy of the tip. The respective voltages of the onset of the valence band $\left(V_{\mathrm{V} \text {,onset }}\right)$ and conduction band $\left(V_{\mathrm{C} \text {,onset }}\right)$ currents times the electron charge $e$ correspond to the position of the valence and conduction band edges $\left(E_{\mathrm{C}}\right)$ relative to the sample Fermi energy $\left(E_{\mathrm{F}} \equiv E_{\mathrm{F} \text {,sample }}\right)$ if flat-band conditions are assumed, as schematically shown in Fig. 3 (a) and (b). This is, however, only valid for systems where the applied voltage between the tip and the sample does not induce a band bending at the sample surface. ${ }^{4}$ In our case, the difference between the onset voltages of the valence and the conduction band-related currents is, however, not constant across the sample surface: In the dark and bright areas, the difference, i.e., ( $V_{\mathrm{C} \text {,onset }}-$ $\left.V_{\mathrm{V} \text {,onset }}\right) \times e$, yields 1.66 and $1.56 \mathrm{eV}$, respectively. Assuming no band bending is present, this would imply that the band gap is apparently fluctuating across the sample surface. However, at the Zn dopant concentrations used, the doping will not modify the bulk band gap of $\mathrm{GaAs}^{42}$ or the surface band structure. Furthermore, the apparent band gap in the tunneling spectra is significantly larger than the real one. Thus, the tip-induced band bending is essential to the quantitative interpretation of the tunneling spectra.

Figure 3 (c) and (d) shows, schematically, how a band bending shifts the band edges if negative and positive voltages are applied to the sample, respectively. In principle, four tunnel currents can occur, as labeled. To discuss these currents, we first turn to the calculated band bending at the GaAs(110) surface as a function of the voltage in Fig. 4. Although the particular calculation is for a carrier concentration of $2 \times 10^{19} \mathrm{~cm}^{-3}$, similar tip-induced band bendings occur for other doping concentrations.

At negative voltages, the tip-induced band bending drags the bands of the semiconductor downward. At voltages large enough, the band bending can in principle pull the conduction band edge below the Fermi energy, and in equilibrium a carrier inversion at the sample surface would form (electrons in the conduction band, as shown in Fig. 3(c)). This inversion 

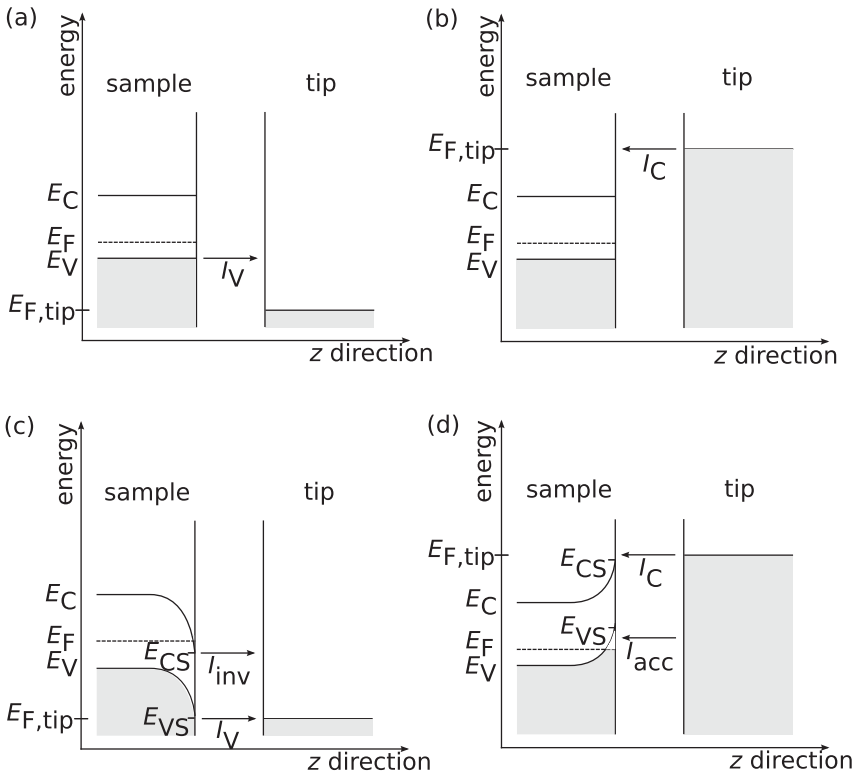

FIG. 3. Schematic illustrations of the band structures of a semiconductor-metal (tip) junction with negative (a and c) and positive ( $b$ and d) voltages applied to the sample. The tip is grounded. (a) and (b) illustrate the flat-band condition without band bending. Currents arising from electrons tunneling from the valence band (a) and into the conduction band (b) can occur. The arrows indicate the tunneling direction. (c) and (d) show the cases including tip-induced downward and upward band bending, respectively. In principle, two additional currents can occur due to electrons tunneling into the accumulation zone (d) or out of the inversion zone (c). All schematics are shown for $p$-type semiconductors. It is assumed that no intrinsic surface states are within the fundamental band gap as for the GaAs(110) surface used here.

is, however, unstable under tunneling conditions because the tunneling rate of these electrons in the conduction band into the metallic tip is much greater than the refilling rate of electrons in the conduction band ${ }^{33}$ due to the negligible free electron concentrations in the conduction band ( $p$-type with holes as majority carriers). A direct excitation over the 1.4-eV-wide band gap or tunneling through the band bending region of $\sim 10 \mathrm{~nm}$ extension (inset of Fig. 4) are both negligible. Thus, no inversion zone and no inversion current $I_{\text {inv }}$ occur, and only electrons tunneling out of the valence band states $\left(I_{\mathrm{V}}\right)$ can be measured at negative voltages. ${ }^{33}$ Therefore, the band bending is only determined by the doping concentration and increases almost linearly with negative voltages.

At positive voltages, the tip induces an upward band bending. Already at small voltages, the valence band edge crosses the Fermi energy on $p$-type surfaces and holes accumulate in the valence band (Fig. 3(d)). These additional holes lead to a much better screening of the tip-induced electric field; thus, the band bending increases slower at positive voltages as compared to negative voltages. In principle, electrons can tunnel into the hole accumulation zone $\left(I_{\text {acc }}\right)$, but the effective barrier is higher than for tunneling into the conduction band $\left(I_{\mathrm{C}}\right)$. Therefore, at positive voltages, the tunnel current into the conduction band dominates.

Finally, the inset in Fig. 4 shows the decay of the band bending into the sample bulk for selected voltages. Within

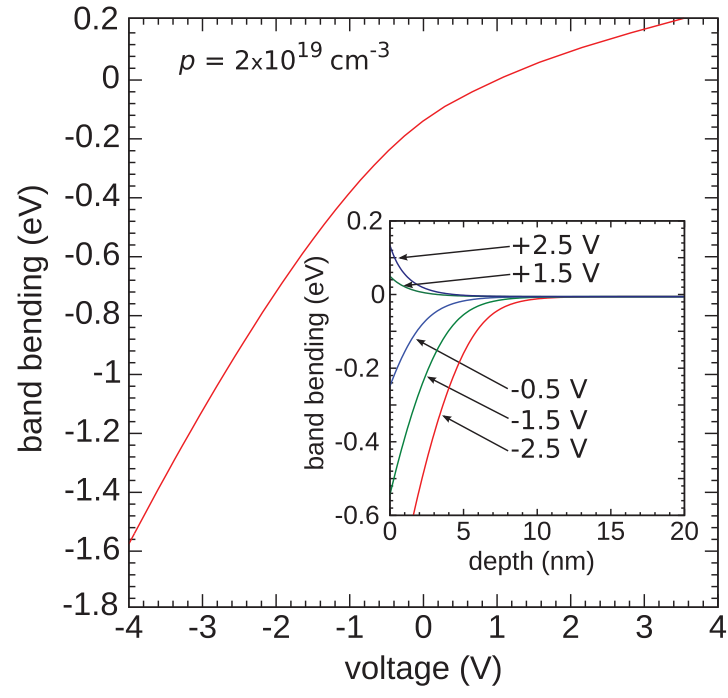

FIG. 4. (Color online) Calculated tip-induced band bending as a function of the voltage applied to the sample. A $p$-type carrier concentration of $2 \times 10^{19} \mathrm{~cm}^{-3}$ was assumed. In the inset, calculated tip-induced band bending is a function of the depth below the semiconductor surface for different voltages, as indicated. The electric field is screened within the first $10 \mathrm{~nm}$. For the calculation, it is assumed that the tip-induced band bending can induce a majority carrier accumulation zone but no inversion zone due to limited carrier dynamics (Ref. 33).

$10 \mathrm{~nm}$, the entire electric field is screened, and thus, the band bending is zero.

In our case, the tip-induced band bending shifts the energy positions of the valence band $\left(E_{\mathrm{VS}}\right)$ and conduction band $\left(E_{\mathrm{CS}}\right)$ edges at the surface underneath the tip. The calculated band edge positions and the Fermi level of the tip $\left(E_{\mathrm{F}, \text { tip }}\right)$ (measured relative to $E_{\mathrm{F} \text {,sample }} \equiv E_{\mathrm{F}} \equiv 0 \mathrm{eV}$ ) are shown in Fig. 5 as a function of the voltage. The Fermi level of the metallic tip is shown as a gray dotted line labeled $E_{\mathrm{F}, \text { tip }}$. It lies in the GaAs band gap at the surface for voltages between +1.4 and $-0.6 \mathrm{~V}$ for the lowest doped sample (labeled $V_{\mathrm{C} \text {,onset }}$ and $V_{\mathrm{V} \text {,onset }}$, respectively). For increasing doping concentrations, the voltage of the valence band onset shifts upward, reaching $-0.3 \mathrm{~V}$ for the highest doped sample shown here. Between the two onset voltages, no electrons can tunnel, because filled (empty) tip states do not face empty (filled) sample states. This 1.7- to 2.0-V-wide voltage region without tunnel current reflects the GaAs band gap of $E_{\text {gap }}=1.4 \mathrm{eV}$. The voltage range times $e$ is larger than the band gap due to the tip-induced band bending, in agreement with the experimental data in Fig. 2. Tunneling out of valence band states is only possible for voltages $V<V_{\mathrm{V} \text {,onset }}$ (note $V$ is negative). In analogy, tunneling into empty conduction band states is only possible for voltages larger than $V_{\mathrm{C} \text {,onset }}$.

At this stage, we address the doping dependence of the onset voltages for electrons tunneling out of the valence and into the conduction bands. This is motivated by the experimental spectra being measured in areas with different local dopant concentrations. Figure 6 shows the calculated onset voltages for the valence and conduction band currents, respectively, as a function of the Fermi level position. The solid line shows an exponential fit to the calculated data points. As expected, 


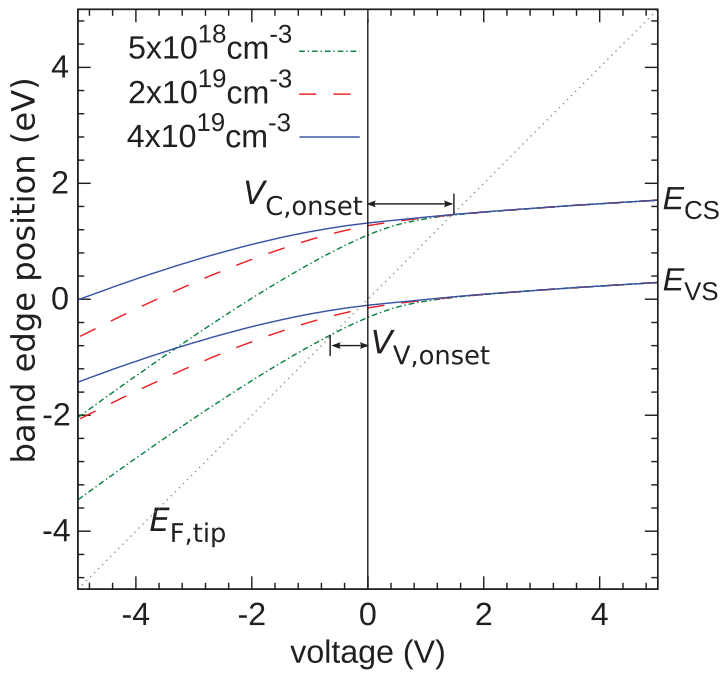

FIG. 5. (Color online) Calculation of the conduction band $\left(E_{\mathrm{CS}}\right)$ and valence band $\left(E_{\mathrm{VS}}\right)$ edge energies at the semiconductor surface of a metal-vacuum-GaAs system as a function of applied sample voltage. Several cases are shown for GaAs with three $p$-type doping levels, as indicated. The Fermi level of the tip $\left(E_{\mathrm{F}, \mathrm{tip}}\right)$ is located at the dotted diagonal line. All energies are given relative to the Fermi level of the sample. The onset voltages for tunneling out of the valence band $\left(V_{\mathrm{V} \text {,onset }}\right)$ and into the conduction band $\left(V_{\mathrm{C} \text {,onset }}\right)$ are indicated for the lowest carrier concentration calculated.

the onset of the valence band current shifts strongly with the doping level, whereas the onset of the conduction band exhibits only a weak change. The difference arises from different screening mechanisms of the electric field between the tip and the sample, as pointed out earlier, i.e., screening by shifting the free holes of the dopant atoms only (negative voltages) or screening by the accumulation of majority carriers in the valence band, whose free hole concentration is dominated by the much larger density of states of the accumulation zone in the valence band and thus essentially independent of the doping concentration (positive voltages). This result agrees with the experimental spectra in Fig. 2 (b)-(d), where the valence band current shifts to smaller absolute values of negative voltages, whereas the conduction band current is almost unchanged. Thus, the experimental data are fully explainable with tip-induced band bending and constant band gap for variable dopant concentrations.

Using the previously presented model, we now address the potential fluctuations. The typical size of the fluctuations of the dopant concentration ranges between 10 and $20 \mathrm{~nm}$ (Fig. 1(a)). The penetration of band bending into the semiconductor bulk is $\sim 10 \mathrm{~nm}$ (inset of Fig. 4). Thus, the decay length of the electric field is smaller than the dimension of the dopant fluctuations. Therefore, we can assume that the STM tip probes a different local dopant concentration in the material independent of the surrounding doping distribution. This can be simulated as if the local dopant cluster is infinitely extended, because the free carriers can fully screen the electric field within the local dopant fluctuation. Only for extremely small dopant agglomerations the screening efficiency is reduced. ${ }^{5}$

To determine the potential from the $I-V$ spectra in Fig. 2(b), we first derive the onset voltages for the valence band currents

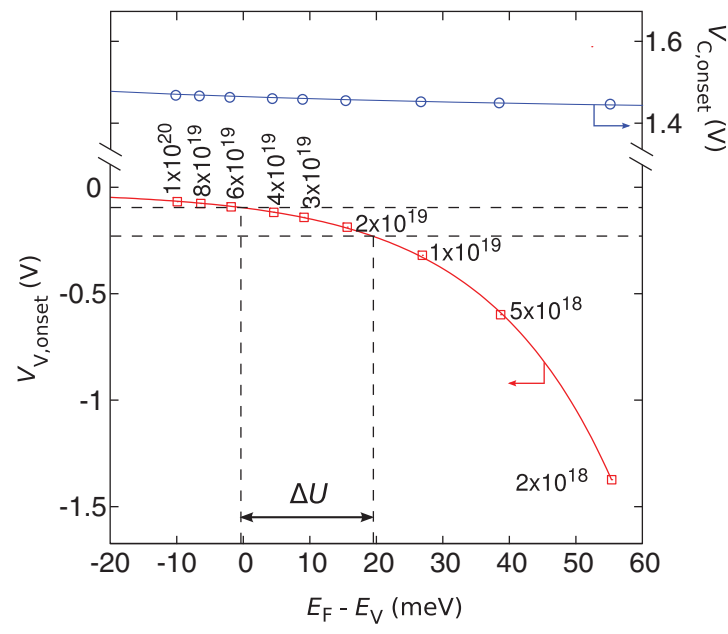

FIG. 6. (Color online) Calculated onset voltages for the tunnel current into the conduction band (top, open circles, $V_{\mathrm{C} \text {,onset }}$ ) and out of the valence band (bottom, open squares, $V_{\mathrm{V} \text {,onset }}$ ) as a function of the Fermi energy relative to the valence band edge and thus the carrier concentration (indicated in units of reciprocal cubic centimeters at each calculated point). The solid lines are fits through the calculated points. The horizontal dashed lines indicate the measured onset voltages of the valence band current for the dark and bright areas. The vertical dashed lines indicate the corresponding Fermi energy positions. The difference is the local potential change between the dark and the bright areas labeled $\Delta U$.

by fitting the experimental data, assuming that the current increases exponentially with increasing absolute voltage starting at the onset voltage. Therefore, we used the following equation $I=I_{0}+a \times \exp \left[b\left(V-V_{\mathrm{V}, \text { onset }}\right)+c\right]$, with $a, b$, and $c$ being constants, to estimate the onset voltages of the valence band currents in Fig. 2(d) (dashed and dotted lines). We obtain for the bright and dark areas' onset voltages of $(-0.086 \pm 0.05)$ and $(-0.226 \pm 0.05) \mathrm{eV}$, respectively. These onset voltages lead through Fig. 6(a) to energy positions of the valence band edge relative to the Fermi level $\left(E_{\mathrm{V}}-E_{\mathrm{F}}\right)$ of about -0.5 and $+19.5 \mathrm{meV}$, respectively (follow the dashed lines). Thus, the potential difference $\Delta U$ is $20 \mathrm{meV}$ between the bright and the dark areas. The particular calculation shown was performed with an effective mass of the valence band density of states of $0.57 m_{0}$ following a recent reassessment of the effective masses. ${ }^{43}$ If we were to use lower values for the effective mass, $\Delta U$ would slightly increase $\left(\sim 1 \mathrm{meV}\right.$ for a $0.1 m_{0}$ change of the effective mass). Thus, the uncertainty of $\Delta U$ arising from the possible error in the effective mass is rather small and can be neglected compared with the error in the determination of the onset voltages. The latter effect induces an error estimated to +20 and $-10 \mathrm{meV}$, i.e., $\Delta U=20_{-10}^{+20} \mathrm{meV}$.

This determination can be corroborated further by determining the typical dopant concentrations in the bright and dark areas. Using the areas marked A and B in Fig. 1(a), we obtain dopant concentration values of $1.5 \times 10^{19}$ and $4.5 \times 10^{19} \mathrm{~cm}^{-3}$ for the dark and bright areas, respectively. These values agree well with the theoretical doping concentrations of $\sim 1.5 \times 10^{19}$ and $5.5 \times 10^{19} \mathrm{~cm}^{-3}$, yielding the measured valence band onset voltages, respectively. This provides further support that the determination of the potential values is correct. 


\section{B. Comparison of dopant-induced potential fluctuations in two- and three-dimensional doping structures}

The potential difference $\Delta U=20 \mathrm{meV}$ between the dark and the bright areas arising from fluctuations in the dopant distribution of three-dimensionally doped GaAs is about one order of magnitude smaller than for potential fluctuations observed in two-dimensional structures $(\Delta U \approx 0.2 \mathrm{eV}$ between the dark and the bright areas). ${ }^{4}$ To explain this difference, we have to recall that in our case the STM can only probe the dopant distribution up to five monolayers below the surface, i.e., $1 \mathrm{~nm}$ (Fig. 1(b)). However, the potential is influenced also by dopant atoms deeper below the surface, because the screening of the local potential by free charge carriers is limited. For the doping concentration used here, the screening length is $1.5 \mathrm{~nm} .^{26,44}$ Thus, dopant atoms within a depth of about two to three times the screening length, i.e., $\sim 5 \mathrm{~nm}$, affect the potential measured at the surface. This effect is damping the potential fluctuations, because we can statistically expect that in the bulk below a surface area with high (low) doping concentration a low (high) dopant atom concentration occurs. In the pure two-dimensional surface doping structure, this damping effect is absent (because no dopants are present below the surface); as a result, larger potential fluctuations occur as measured. ${ }^{4}$ In addition, the screening length in two-dimensional systems is shorter, ${ }^{26}$ which again reduces any damping of the potential fluctuations.

\section{Implications for quantitative band offset and band gap measurements}

At this stage, we address the issue of extracting band offset values and band gaps in cross-sectional scanning tunneling spectra of compound semiconductor surfaces. The band bending effects discussed earlier are detrimental to quantitative band edge energy determinations by scanning tunneling spectroscopy, because significant shifts of the onset voltages occur even at rather high doping concentrations if the surfaces are unpinned. Thus, for unpinned surfaces, it is unavoidable to take the band bending effects into account using the methodology presented previously. The tip-induced band bending can be experimentally reduced by using extremely high doped materials (in the range of $10^{20}$ $\mathrm{cm}^{-3}$ ) or an extremely sharp tip. ${ }^{45}$ However, even then we need to determine and address the effect of the tip-induced band bending on the quantitative values extracted.

In case of pinned surfaces, the band edge position can be determined exactly, because no tip-induced band bending occurs. However, in this case, the band edge positions measured at the surface are not representative of those in the bulk. Instead, the band offsets of heterojunctions are modified by the specific location of the surface pinning levels in the different materials (e.g., AlGaAs and GaAs). On the other hand, the determination of the band gap is then highly accurate, because no apparent enlargement of the band gap occurs due to tip-induced band bending. ${ }^{41,46}$ Thus, the procedure of band gap determination is much simpler than for unpinned surfaces discussed in Ref. 33. A pinning can be achieved by introducing localized states in the fundamental band gap at the surface. This can be done, e.g., by thermally producing point defects, such as vacancies, ${ }^{47}$ by cleavages with high step densities ${ }^{46}$ or by adsorption of atoms or molecules (either intentionally or unintentionally). In any case, it is crucial to know exactly what is in which concentration present on the surface. Simple measurement without determinations of the state of the surface provides little quantitative insight.

\section{CONCLUSIONS}

Local potential fluctuations induced by nanoscale inhomogeneities in the three-dimensional spatial dopant distribution in Zn-doped GaAs were investigated quantitatively by STM and spectroscopy on (110) cleavage surfaces. Tunneling spectra measured in areas with different local doping concentrations showed apparent shifts of the valence band edge and apparent changes of the band gap. A quantitative analysis, combined with band bending and tunnel current simulations, demonstrated that these effects arise from tip-induced band bending that modulates the real potential changes. A methodology was illustrated on how the exact potential difference between locally high and low doped areas can be determined. It was found that the potential varies between high and low doped areas by $20 \mathrm{meV}$. These local potential fluctuations in threedimensionally doped semiconductors were approximately one order of magnitude smaller than those observed in twodimensionally doped semiconductors. This was explained by damping effects due to the three-dimensional arrangement of the dopants, which are absent in purely two dimensionally doped semiconductors or semiconducting structures.

This effect may have a significant impact on the device properties if the miniaturization is coupled with dimensionally reduced structures, e.g., purely two-dimensional channels in transistors, because the potential fluctuations due to dopant inhomogeneities may increase by one order of magnitude in two-dimensional versus three-dimensional structures, assuming no pinning effect of surrounding semiconductor interfaces/surfaces. We speculate that the dopant-induced potential fluctuations increase even further if one-dimensional channels are used.

\section{ACKNOWLEDGMENT}

The authors thank K. Urban and J. Mayer for helpful discussions and support, M. Yurechko for participation in initial measurements, $H$. Eisele for critically reading the manuscript, and K. H. Graf for technical support.

\footnotetext{
*p.ebert@fz-juelich.de

${ }^{1}$ S. Roy and A. Asenov, Science 309, 388 (2005).

${ }^{2}$ M. R. Castell, D. A. Muller, and P. M. Voyles, Nat. Mater. 2, 129 (2003).
}

${ }^{3}$ Ph. Ebert, T. Zhang, F. Kluge, M. Simon, Z. Zhang, and K. Urban, Phys. Rev. Lett. 83, 757 (1999).

${ }^{4}$ S. Landrock, Y. Jiang, K. H. Wu, E. G. Wang, K. Urban, and Ph. Ebert, Appl. Phys. Lett. 95, 072107 (2009). 
${ }^{5}$ N. D. Jäger, K. Urban, E. R. Weber, and Ph. Ebert, Appl. Phys. Lett. 82, 2700 (2003).

${ }^{6}$ W. D. Rau, P. Schwander, F. H. Baumann, W. Höppner, and A. Ourmazd, Phys. Rev. Lett. 82, 2614 (1999).

${ }^{7}$ A. C. Twitchett, R. E. Dunin-Borkowski, and P. A. Midgley, Phys. Rev. Lett. 88, 238302 (2002).

${ }^{8}$ N. Ikarashi, T. Ikezawa, K. Uejima, T. Fukai, M. Miyamura, A. Toda, and M. Hane, J. Appl. Phys. 103, 114514 (2008).

${ }^{9}$ D. Cooper, J.-M. Hartmann, B. Aventurier, F. Templier, and A. Chabli, Appl. Phys. Lett. 93, 183509 (2008).

${ }^{10}$ A. C. Twitchett-Harrison, T. J. V. Yates, S. B. Newcomb, R. E. Dunin-Borkowski, and P. A. Midgley, Nano Lett. 7, 2020 (2007).

${ }^{11}$ A. C. Twitchett-Harrison, T. J. V. Yates, R. E. Dunin-Borkowski, and P. A. Midgley, Ultramicroscopy 108, 1401 (2008).

${ }^{12}$ P. M. Voyles, D. A. Muller, J. L. Grazul, P. H. Citrin, and H. J. Gossmann, Nature 416, 826 (2002).

${ }^{13}$ Y. Rosenwaks, R. Shikler, Th. Glatzel, and S. Sadewasser, Phys. Rev. B 70, 085320 (2004).

${ }^{14}$ H. W. M. Salemink, O. Albrektsen, and P. Koenraad, Phys. Rev. B 45, 6946 (1992).

${ }^{15}$ Y. Dong, R. M. Feenstra, R. Hey, and K. H. Ploog, J. Vac. Sci. Technol. B 20, 1677 (2002).

${ }^{16}$ N. D. Jäger, K. Urban, E. R. Weber, and Ph. Ebert, Phys. Rev. B 65, 235302 (2002).

${ }^{17}$ R. J. Hamers, J. Vac. Sci. Technol. B 6, 1462 (1988).

${ }^{18}$ J. A. Stroscio, R. M. Feenstra, and A. P. Fein, Phys. Rev. Lett. 58, 1668 (1987).

${ }^{19} \mathrm{Ph}$. Ebert, X. Chen, M. Heinrich, M. Simon, K. Urban, and M. G. Lagally, Phys. Rev. Lett. 76, 2089 (1996).

${ }^{20} \mathrm{M}$. Heinrich, C. Domke, Ph. Ebert, and K. Urban, Phys. Rev. B 53, 10894 (1996).

${ }^{21}$ C. Domke, M. Heinrich, Ph. Ebert, and K. Urban, J. Vac. Sci. Technol. B 16, 2825 (1998).

${ }^{22}$ S. Perraud, K. Kanisawa, Z.-Z. Wang, and T. Fujisawa, Phys. Rev. B 76, 195333 (2007).

${ }^{23}$ M. Morgenstern, J. Klijn, C. Meyer, M. Getzlaff, R. Adelung, R. A. Römer, K. Rossnagel, L. Kipp, M. Skibowski, and R. Wiesendanger, Phys. Rev. Lett. 89, 136806 (2002).

${ }^{24}$ S. Modesti, D. Furlanetto, M. Piccin, S. Rubini, and A. Franciosi, Appl. Phys. Lett. 82, 1932 (2003).

${ }^{25}$ N. D. Jäger, M. Marso, M. Salmeron, E. R. Weber, K. Urban, and Ph. Ebert, Phys. Rev. B 67, 165307 (2003).

${ }^{26}$ A. Laubsch, K. Urban, and Ph. Ebert, Phys. Rev. B 80, 245314 (2009).

${ }^{27}$ S. Yoshida, Y. Kanitani, A. Takeuchi, and H. Shigekawa, Appl. Phys. Lett. 92, 102105 (2008).

${ }^{28}$ R. M. Feenstra and J. A. Stroscio, J. Vac. Sci. Technol. B 5, 923 (1987).

${ }^{29}$ M. McEllistrem, G. Haase, D. Chen, R. J. Hamers, Phys. Rev. Lett. 70, 2471 (1993).

${ }^{30}$ R. Dombrowski, C. Steinebach, C. Wittneven, M. Morgenstern, and R. Wiesendanger, Phys. Rev. B 59, 8043 (1999).

${ }^{31}$ M. Wenderoth, M. A. Rosentreter, A. J. Heinrich, M. A. Schneider, and R. G. Ulbrich, Euro. Phys. Lett. 45, 579 (1999).

${ }^{32}$ R. Seiwatz and M. Green, J. Appl. Phys. 29, 1034 (1958).
${ }^{33}$ N. D. Jäger, E. R. Weber, K. Urban, and Ph. Ebert, Phys. Rev. B 67, 165327 (2003).

${ }^{34}$ J. Bono and R. H. Good Jr., Surf. Sci. 175, 415 (1986).

${ }^{35}$ J. Tersoff and D. R. Hamann, Phys. Rev. B 31, 805 (1985).

${ }^{36}$ A. Selloni, P. Carnevali, E. Tosatti, and C. D. Chen, Phys. Rev. B 31, 2602 (1985).

${ }^{37}$ R. M. Feenstra, J. A. Stroscio, J. Tersoff, and A. P. Fein, Phys. Rev. Lett. 58, 1192 (1987); Ph. Ebert, B. Engels, P. Richard, K. Schroeder, S. Blügel, C. Domke, M. Heinrich, and K. Urban, Phys. Rev. Lett. 77, 2997 (1996); B. Engels, P. Richard, K. Schroeder, S. Blügel, Ph. Ebert, and K. Urban, Phys. Rev. B 58, 7799 (1998); Ph. Ebert, G. Cox, U. Poppe, and K. Urban, Surf. Sci. 271, 587 (1992); Ph. Ebert, G. Cox, U. Poppe, and K. Urban, Ultramicroscopy 42-44, 871 (1992).

${ }^{38}$ G. Lengel, R. Wilkins, G. Brown, M. Weimer, J. Gryko, and R. E. Allen, Phys. Rev. Lett. 72, 836 (1994); Ph. Ebert, K. Urban, and M. G. Lagally, Phys. Rev. Lett. 72, 840 (1994); Ph. Ebert, K. Urban, L. Aballe, C. H. Chen, K. Horn, G. Schwarz, J. Neugebauer, and M. Scheffler, Phys. Rev. Lett. 84, 5816 (2000).

${ }^{39} \mathrm{Ph}$. Ebert, Surf. Sci. Rep. 33, 121 (1999); Ph. Ebert, Curr. Opin. Solid State Mater. Sci. 5, 211 (2001).

${ }^{40}$ M. B. Johnson, O. Albrektsen, R. M. Feenstra, and H. W. M. Salemink, Appl. Phys. Lett. 63, 2923 (1993); 64, 1454 (1994); R. M. Feenstra, E. T. Yu, J. M. Woodall, P. D. Kirchner, C. L. Lin, and G. D. Pettit, Appl. Phys. Lett 61, 795 (1992); $\mathrm{Ph}$. Ebert and K. Urban, Ultramicroscopy 49, 344 (1993); J. F. Zheng, X. Liu, N. Newman, E. R. Weber, D. F. Ogletree, and M. Salmeron, Phys. Rev. Lett. 72, 1490 (1994); Ph. Ebert, M. Heinrich, M. Simon, C. Domke, K. Urban, C.-K. Shih, M. B. Webb, and M. G. Lagally, Phys. Rev. B 53, 4580 (1996); C. Domke, Ph. Ebert, and K. Urban, Surf. Sci. 415, 285 (1998); G. Mahieu, B. Grandidier, D. Deresmes, J. P. Nys, D. Stiévenard, and Ph. Ebert, Phys. Rev. Lett. 94, 026407 (2005).

${ }^{41} \mathrm{Ph}$. Ebert, S. Schaafhausen, A. Lenz, A. Sabitova, L. Ivanova, M. Dähne, Y.-L. Hong, S. Gwo, and H. Eisele, Appl. Phys. Lett. 98, 062103 (2011).

${ }^{42}$ The covalent radii of $\mathrm{Zn}$ and $\mathrm{Ga}$ of 1.25 and $1.26 \AA$, respectively, are almost identical. Therefore, no strain is expected for $\mathrm{Zn}$ dopants replacing $\mathrm{Ga}$ atoms in GaAs. Indeed, thus far no $\mathrm{Zn}$-dopant-induced strain effects were reported in GaAs, in contrast to, e.g., Si dopant atoms in $\mathrm{GaN}$, where strain effects in modulation-doped material can be observed by cross-sectional STM [H. Eisele, L. Ivanova, S. Borisova, M. Dähne, M. Winkelnkemper, and Ph. Ebert, Appl. Phys. Lett. 94, 162110 (2009)].

${ }^{43}$ S. Adachi, GaAs and Related Materials: Bulk Semiconducting and Superlattice Properties (World Scientific, Singapore, 1994) p. 254.

${ }^{44}$ R. B. Dingle, Philos. Mag. 46, 831 (1955).

${ }^{45}$ R. M. Feenstra, Phys. Rev. B 50, 4561 (1994).

${ }^{46}$ L. Ivanova, S. Borisova, H. Eisele, M. Dähne, A. Laubsch, and Ph. Ebert, Appl. Phys. Lett. 93, 192110 (2008); Ph. Ebert, L. Ivanova, and H. Eisele, Phys. Rev. B 80, 085316 (2009).

${ }^{47} \mathrm{Ph}$. Ebert, M. Heinrich, M. Simon, K. Urban, and M. G. Lagally, Phys. Rev. B 51, 9696 (1995); M. Heinrich, Ph. Ebert, M. Simon, K. Urban, and M. G. Lagally, J. Vac. Sci. Technol. A 13, 1714 (1995). 